\begin{tabular}{|c|c|}
\hline Title & The effect of microstructure on very high cy cle fatigue properties in Ti-6A I- $4 \mathrm{~V}$ \\
\hline Author(s) & Oguma, H.; Nakamura, T. \\
\hline Citation & $\begin{array}{l}\text { Scripta Materialia, 63(1), 32-34 } \\
\text { https://doi.org/10.1016/.scriptamat.2010.02.043 }\end{array}$ \\
\hline Issue Date & $2010-07$ \\
\hline Doc URL & http:/hdl.handle.net/2115/43173 \\
\hline Type & article (author version) \\
\hline File Information & SM63-1_32-34.pdf \\
\hline
\end{tabular}

Instructions for use 


\title{
The effect of microstructure on very high cycle fatigue properties in Ti-6Al-4V
}

\author{
H. Oguma ${ }^{\mathrm{a},{ }^{*}}$ and T. Nakamura ${ }^{\mathrm{a}}$ \\ ${ }^{a}$ Div. of Mech. \& Space Eng., Hokkaido University, Sapporo, 060-8628 Japan
}

Uniaxial tension fatigue tests were conducted on a Ti-6Al-4V alloy that underwent different heat treatments to reveal the effect of microstructures on the very high cycle fatigue properties. The size dependency of threshold stress intensity factor range will be pointed out for the sub-surface crack, and a possible mechanism will be presented for the phenomenon that the sub-surface fractures occur at lower stress levels in the very high cycle regime than do surface-initiated fractures.

Keywords: High cycle fatigue; Titanium; Microstructure; Sub-surface fracture

In recent years the very high cycle fatigue (VHCF) of high strength material has become a topic of great interest for many advanced structural designs. In the lower fatigue life region, the origin of fatigue fracture is usually at the surface, whereas for longer fatigue lifetimes origins are generally sub-surface in nature. It has been reported that the subsurface fractures occur at a lower stress level than the surface-initiated fracture [1]. As yet there is not a clear understanding of the mechanism that is responsible for the fatigue process in VHCF. Regarding Ti-6Al-4V alloy, a commonly used turbine blade alloy, the effect of microstructure on fatigue crack initiation and propagation has been widely studied from experimental viewpoints [2, 3]. As for VHCF properties, however, the role of microstructures has not been studied systematically. From this point of view, uniaxial tension fatigue tests were carried out on $(\alpha+\beta)$ Ti-6Al-4V alloy that underwent different heat treatments to reveal the effect of microstructure on the VHCF properties. Additionally the size dependency of threshold stress intensity factor range will be pointed out for the sub-surface crack, and a possible mechanism for the phenomenon that subsurface fractures occur at lower stress levels than surface-initiated fractures will be presented.

Two forms of material were used. One was a circular rod of diameter of $16 \mathrm{~mm}$, which underwent the following process: Forging $\rightarrow$ Rolling (1223K) $\rightarrow$ Air-cooled annealing (993 1013K, $3.6 \mathrm{ks}$ ). The other was a circular rod of diameter of $20 \mathrm{~mm}$, which was made by Gyratory Forging Machine (GFM), given the heat treatment of 1203K, 3.6 ks, $\mathrm{AC} \rightarrow 978 \mathrm{~K}, 7.2 \mathrm{ks}$, AC. In this study, the former material is designated as MA for MillAnnealed and the latter as STOA for Solution heat Treatment and Over Aged. The microstructures of these materials after the heat treatment are dual phase with $(\alpha+\beta)$, and the average size of the $\alpha$ grain is $4 \mu \mathrm{m}$ for MA and $10 \mu \mathrm{m}$ for STOA. Static strengths of these materials are tensile strength of $988 \mathrm{MPa}$ (MA), $943 \mathrm{MPa}$ (STOA) and yield strength of $899 \mathrm{MPa}$ (MA), $860 \mathrm{MPa}$ (STOA) respectively. After lathe turning these materials into an hourglass-type test piece of $4.1 \mathrm{~mm}$ minimum diameter, each specimen was polished with \#120 to \#1500 emery paper. Uniaxial tension fatigue tests were carried out under sinusoidal waveform loading at a stress ratio of 0.1 at room temperature using an electro-servo-hydraulic-testing machine at $120 \mathrm{~Hz}$. 
The number of cycles to failure $\mathrm{N}_{\mathrm{f}}$ as a function of maximum stress $\sigma_{\max }$ is shown in Figure 1. With the surface-initiated and sub-surface fractures, the S-N curves of both materials are similar, gentle slopes at high stresses and steep inclinations at lower stresses. In the case of negative stress ratios, the $\mathrm{S}-\mathrm{N}$ curves of these materials show the general feature of monotonic increases in fatigue life with decreasing stress [4]. Therefore, the steep inclinations at lower stresses are a remarkable characteristic under the positive stress ratio. The test data for the MA display superior VHCF properties, with higher fatigue strengths of both surface and sub-surface fractures. Only in STOA, interference type fractures were observed where the main crack was formed by a coalescence of surface-initiated and sub-surface initiated cracks. These tendencies may result from a higher resistance to crack initiation as well as to crack propagation of the MA microstructure.

The fracture surface observation using a scanning electron microscope (SEM) has revealed that fatigue cracks propagated from plane facets, and the facet is confirmed as transgranular cracking of an $\alpha$ grain [4]. The relationship between fatigue strength and $\alpha$ grain size is shown in Figure 2, and the tensile and yield strengths are also indicated in the figure. The fatigue strength at $10^{6}$ cycles was defined as the average of the stress of censored data at $10^{6}$ cycles and the minimum stress among the fracture data below $10^{6}$ cycles. The fatigue strength at $10^{8}$ cycles was calculated using the same definition. All strengths show a similar behavior, decreasing with increasing grain size. The differences in tensile strength, yield strength and fatigue strength at $10^{6}$ cycles are very similar, about $50 \mathrm{MPa}$. Meanwhile, the difference in the fatigue strength is about $170 \mathrm{MPa}$ and three times greater at $10^{8}$ cycles than at $10^{6}$ cycles. The finer microstructure of MA material improved the fatigue strength in VHCF regime. When the $\alpha$ grain size becomes smaller, a commensurate reduction in effective slip length and corresponding increase in yield strength are observed. In addition, the sizes of the $\alpha$ grains strongly correlate with the occurrence of sub-surface fractures as compared to surface-initiated fractures.

It is generally known that the threshold stress intensity factor range $\left(\Delta K_{\text {th }}\right)$ of large crack is determined by the material, stress ratio and environment. In small cracks, however, the $\Delta K_{\mathrm{th}}$ is determined by both the material and by the size of the crack. The smaller the crack length, the lower the $\Delta K_{\text {th }}$ becomes, and the crack closure stress decreases due to reductions in the area of the crack surface needed for crack closure [5]. The $\Delta K_{\text {th }}$ of large cracks is typically a constant value and larger than $\Delta K_{\text {th }}$ of small cracks. The relation between crack length and $\Delta K_{\text {th }}$ in the Ti-6Al-4V alloy was investigated by Nakamura et al. [6], with uniaxial tension fatigue tests in air and in vacuum using test specimens of STOA with small artificial defects. The relation obtained in the study is shown in Figure 3. In the figure, the crack size is evaluated by $\sqrt{ }$ area (the area is the projected area of the crack on the principal stress plane). The tendency towards smaller $\Delta K_{\text {th }}$ with decreasing crack size occurs both in air and in vacuum. The tendency towards smaller $\Delta K_{\text {th }}$ was stronger in vacuum than in air, and the inclination of the line for vacuum is steeper than that for air, suggesting that differences in environments affect the size dependency of the $\Delta K_{\mathrm{th}}$. The effect of the oxide-induced crack closure and the plasticity-induced crack closure was suggested to explain this difference of the size dependencies. As a result, $\Delta K_{\text {th }}$ values in 
vacuum would have smaller values than in air when the crack lengths are comparable to the $\alpha$ grain size of $\sim 10 \mu \mathrm{m}$.

To investigate the size dependence of $\Delta K_{\text {th }}$ on sub-surface cracks, the test data of the subsurface fractures and unbroken specimens in Figure 1 were rearranged by the initial stress intensity factor range $\left(\Delta K_{\text {ini }}\right)$ as shown in Figure 4 . The $\Delta K_{\text {ini }}$ were calculated from the following equation [7];

$$
\Delta K_{\text {ini }}=0.5 \Delta \sigma \sqrt{\pi \sqrt{\text { area }}} \quad\left(\Delta \sigma=\sigma_{\max }-\sigma_{\min }\right)
$$

where the average $\alpha$ grain sizes, $4 \mu \mathrm{m}$ for MA and $10 \mu \mathrm{m}$ for STOA, were substituted into the Varea as the initial crack lengths. In the Ti-6Al-4V alloy, the fatigue limit of the subsurface fracture was caused by the non-propagation of the crack [4]. Consequently, $\Delta K_{\text {th }}$ corresponds to the lower limit of $\Delta K_{\text {ini }}$ in Figure 4. To determine $\Delta K_{\text {th }}$, the average of the minimum value of $\Delta K_{\text {ini }}$ of the sub-surface fractures and the maximum value among $\Delta K_{\text {ini }}$ of the unbroken specimens were calculated. The $\Delta K_{\text {th }}$ of MA and STOA are $1.08 \mathrm{MPa} \sqrt{\mathrm{m}}$ and $1.45 \mathrm{MPa} \vee \mathrm{m}$, respectively. The $\Delta K_{\text {th }}$ of MA is, therefore, smaller than that of STOA in the interior fractures. This result indicates a crack size dependence of $\Delta K_{\text {th }}$ in the subsurface crack propagation of this material.

The similarities in the fracture surfaces for sub-surface fracture and surface fractures in vacuum were discussed in a previous study [6]. It was suggested that the effect of the interior environment of the material and that of vacuum on the crack propagation are very similar. The dependence of $\Delta K_{\text {th }}$ on crack size was different in air and in vacuum, as shown in Figure 3. A size dependence of $\Delta K_{\text {th }}$ was also observed in the sub-surface cracks. There is a very strong possibility that the $\Delta K_{\text {th }}$ in the sub-surface environment becomes smaller than that in air when the size of the crack length is comparable to that of the fracture origin, the $\alpha$ grain(s) in Ti-6Al-4V. Such a hypothesis suggests that sub-surface cracks may propagate and lead to fracture under conditions where a surface crack cannot propagate. The size dependence of $\Delta K_{\text {th }}$ depends on the environment around the fatigue crack tip, and the dependence on the environment can explain why the sub-surface fractures occur at lower stress levels than surface-initiated fractures.

It is concluded that the miniaturization of microstructure improved well the fatigue strength in VHCF regime in Ti-6Al-4V alloy. The sizes of the $\alpha$ grains strongly affect the occurrence of the sub-surface fractures than that of the surface-initiated fractures. The crack size dependence of $\Delta K_{\text {th }}$ in the sub-surface fracture was observed. The occurrence of the sub-surface fractures at lower stress levels than the surface-initiated fractures can be accounted for the size dependency.

[1] S. Nishijima, K. Kanazawa, Fatigue Fract. Engng. Mater. Struct., 22 (1999) 601. [2] R. K. Nalla, B. L. Boyce, J. P. Campbell, J. O. Peters, R. O. Ritchie, Metallurgical and Materials Transactions, 33A (2002) 899.

[3] K. Tokaji, J. C. Bian, T. Ogawa, M. Nakajima, Material Science and Engineering, A213 (1996) 86.

[4] H. Oguma, PhD Thesis, Hokkaido University, 2006. 
[5] Y. Murakami, T. Nomoto and T. Ueda, Fatigue Fract Engng. Mater. Struct., 22 (1999) 581.

[6] T. Nakamura, T. Shiina, S. Yokoyama and T. Noguchi, Proc. $3^{\text {rd }}$ Int. Conf. VHCF-3 (2004) 569.

[7] Y. Murakami, Eng. Fract. Mech., 22 (1985) 101.

*Corresponding author.

Tel.: +81 (0)11 706 6421; e-mail: Hiroyuki.Oguma@eng.hokudai.ac.jp

Figure 1. S-N curves.

Figure 2. Relation between fatigue strength and $\alpha$ grain size with tensile and yield strengths shown for reference.

Figure 3. Relation between threshold stress intensity factor range and defect size (Ti$6 \mathrm{Al}-4 \mathrm{~V}, \mathrm{STOA})[6]$.

Figure 4. Relation between the stress intensity factor range of origin and fatigue life. 


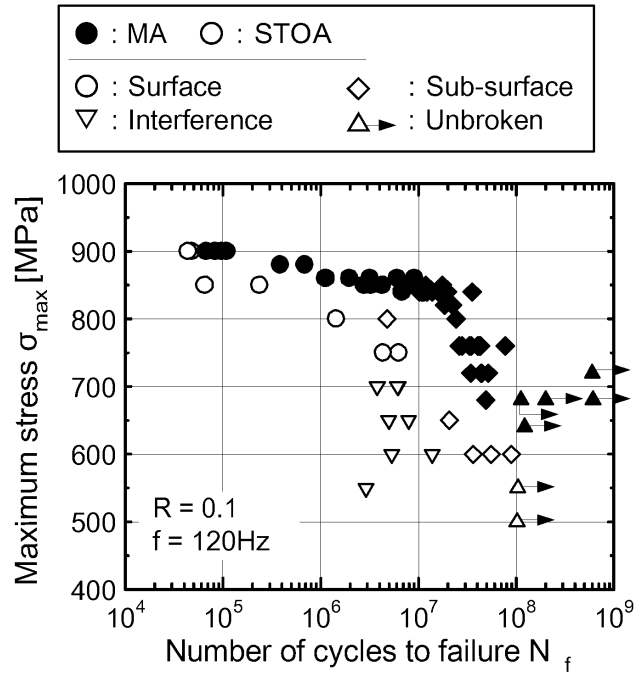

Figure 1. S-N curves.

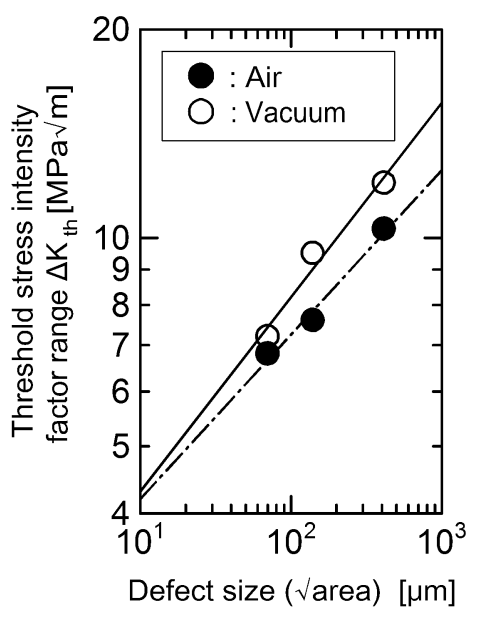

Figure 3. Relation between threshold stress intensity factor range and defect size (Ti-6Al-4V, STOA) [6].

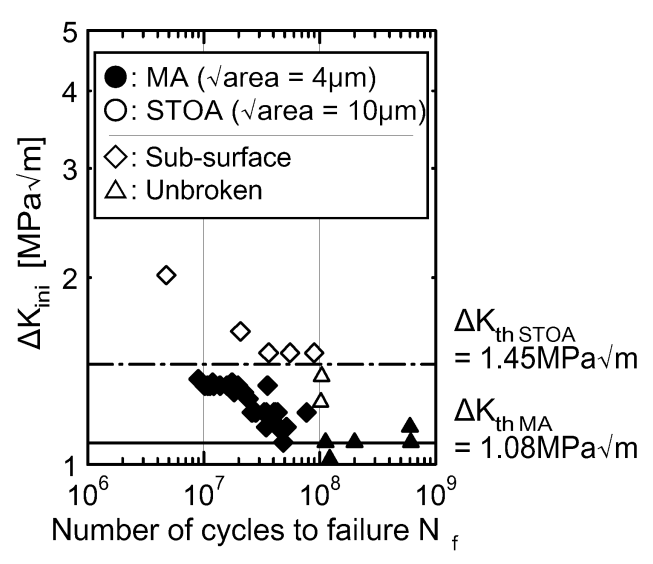

Figure 4. Relation between the stress intensity factor range of origin and fatigue life. 